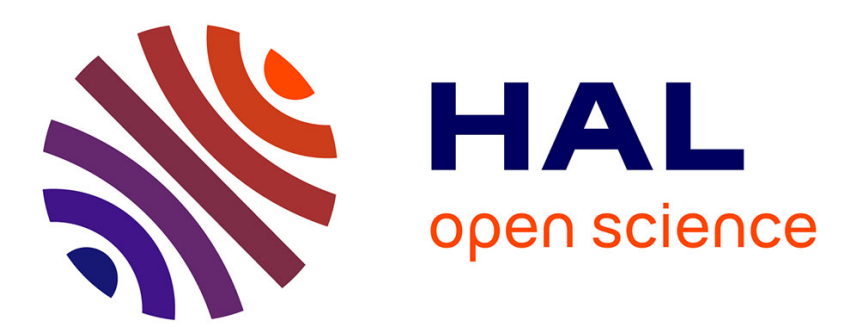

\title{
Analyse numérique du fonctionnement des structures lasers à cavités couplées
}

L. Vassilieff, A. Bensoussan, Françoise Lozes-Dupuy, H. Martinot, G. Vassilieff

\section{To cite this version:}

L. Vassilieff, A. Bensoussan, Françoise Lozes-Dupuy, H. Martinot, G. Vassilieff. Analyse numérique du fonctionnement des structures lasers à cavités couplées. Revue de Physique Appliquée, 1987, 22 (8), pp.919-930. 10.1051/rphysap:01987002208091900 — jpa-00245633

\section{HAL Id: jpa-00245633 https://hal.science/jpa-00245633}

Submitted on 1 Jan 1987

HAL is a multi-disciplinary open access archive for the deposit and dissemination of scientific research documents, whether they are published or not. The documents may come from teaching and research institutions in France or abroad, or from public or private research centers.
L'archive ouverte pluridisciplinaire HAL, est destinée au dépôt et à la diffusion de documents scientifiques de niveau recherche, publiés ou non, émanant des établissements d'enseignement et de recherche français ou étrangers, des laboratoires publics ou privés. 
Classification

Physics Abstracts

$42.55 \mathrm{P}-42.60$

\title{
Analyse numérique du fonctionnement des structures lasers à cavités couplées
}

\author{
L. Vassilieff, A. Bensoussan, F. Lozes-Dupuy, H. Martinot et G. Vassilieff \\ Laboratoire d'Automatique et d'Analyse des Systèmes du C.N.R.S., 7, avenue du Colonel Roche, \\ 31077 Toulouse Cedex, France
}

(Reçu le 2 février 1987, révisé le 24 mars 1987, accepté le 9 avril 1987)

\begin{abstract}
Résumé. - Nous présentons ici une analyse numérique des structures lasers à cavités couplées, permettant d'obtenir les modes longitudinaux admis par la structure et le déplacement du pic laser en fonction des conditions de polarisation. La valeur absolue de la puisance optique sur chaque miroir est obtenue en traitant la structure à 2 cavités au moyen du concept d'amplificateur résonant. Les résultats obtenus constituent un guide dans l'utilisation de ces composants en fonction du résultat désiré. Ils montrent en particulier dans quelles conditions la cavité couplée est particulièrement accordable en fréquence ou, à l'opposé, peut être maintenue à une longueur d'onde stable en fonction des courants et des tensions appliqués.
\end{abstract}

\begin{abstract}
A numerical analysis of coupled cavity lasers is given which permits the determination of the longitudinal modes of the structure and of the emitted peak as function of the bias level. On the basis of the resonance amplifier model, the optical power absolute value on each mirror is calculated. The results of this simulation constitute a guide in the utilisation of the device according to the desired application. Particularly they show in which conditions the coupled cavity is the most tunable or, on the contrary, can be maintained monomode stable versus the applied currents and bias level.
\end{abstract}

\section{Introduction.}

Les structures lasers à cavités couplées présentent par rapport aux lasers classiques les avantages suivants :

- spectre d'émission monomode longitudinal,

- longueur d'onde ajustable par voie électrique,

- commutation en fréquence et bistabilité optique,

- diversité de comportement suivant le type de sillon réalisé,

- photodiode de contrôle intégrée,

- comportement en «transistor laser » récemment publié [1].

Leur utilisation s'accroît dans le domaine industriel, soit dans le but de stabiliser la longueur d'onde d'émission laser et d'en affiner la raie, soit en tant que source laser accordable en fréquence dans les systèmes à plusieurs longueurs d'ondes.

Nous présenterons ici un modèle théorique permettant de décrire de façon exhaustive le fonctionnement de ces composants en régime statique. Ce modèle appliqué au cas du GaAlAs permet d'expli- quer et de coordonner les différents comportements expérimentaux décrits dans la littérature [2-4].

La structure de base du laser à cavités couplées est représentée sur la figure 1. Le sillon séparant les deux cavités est généralement réalisé par «R.I.E. » (Reactive Ion Etching) et conduit à des comporte-

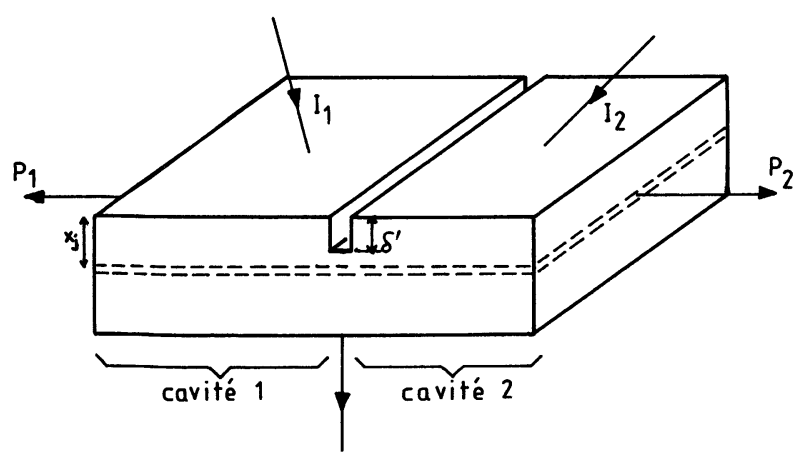

Fig. 1. - Schéma général d'une structure laser à cavités couplées.

[Schematic drawing of coupled cavity lasers.] 
ments différents selon sa profondeur, d'une part et le pouvoir de réflexion de ses flancs d'autre part :

si $\delta^{\prime}>x_{\mathrm{j}} \rightarrow$ comportement de type « $\mathrm{C}^{3}$ » (cleaved coupled cavity [16]),

si $\delta^{\prime}<x_{j} \rightarrow$ comportement de type API ou IRI (active passive interference [22], or internal reflection interference [23] lasers),

$x_{\mathrm{j}}$ étant la profondeur de jonction par rapport à la surface,

$\delta$ ' étant la profondeur du sillon séparant les deux cavités.

\section{Analyse.}

L'analyse théorique est menée sur la base du Resonance Amplifier Model $[5,6]$ en prenant en compte la répartition de l'émission spontanée sur chaque mode laser, l'effet de la densité de porteurs sur l'indice de zone active, et le traitement de l'interaction optique entre les deux cavités par le formalisme de la matrice « $S$ » (Scattering matrix [7]). Son application est faite dans le cas du GaAlAs.

\section{Hypothèses :}

- constante de propagation complexe indépendante de la distance $z$ sur l'axe de propagation dans chaque cavité,

- coefficient d'absorption $\alpha^{\prime}$ pris constant en fonction de la densité de porteurs injectée dans la zone active, la valeur choisie correspond à $N=$ $10^{18} \mathrm{~cm}^{-3}$ caractéristique de la densité de porteurs en fonctionnement laser dans la zone active, réels,

- coefficients $S_{i j}$ de la matrice « $S$ » supposés

- propagation du seul mode transverse fondamental.

La détermination des grandeurs intervenant dans la réponse optique du dispositif nécessite :

- la connaissance de la densité de porteurs dans la zone active en GaAlAs : celle-ci est calculée en fonction de l'écart $\Delta E_{\mathrm{F}}$ des pseudo-niveaux de Fermi, par la statistique de Fermi appliquée aux trois bandes de conductions $\Gamma, X$ et $L$ de ce matériau, compte tenu de la température et de sa composition en aluminium [8] ;

- la prise en compte de l'influence de la densité de porteurs injectée dans la zone active sur l'indice de réfraction optique.

L'indice de zone active, en fonction de $x_{\mathrm{Al}}, T, \lambda$ et $N$, est alors exprimé par :

$$
\begin{aligned}
n_{\mathrm{act}}=n_{0}+a_{x_{\mathrm{Al}}} x_{\mathrm{Al}} & +a_{\lambda}\left(\lambda-\lambda_{0}\right)+ \\
& +a_{N}\left(N-N_{0}\right)+a_{\mathrm{T}}\left(T-T_{0}\right)
\end{aligned}
$$

avec

$$
\begin{gathered}
a_{x_{\mathrm{Al}}}=-0,62[9] \quad a_{\lambda}=-10^{6} \mathrm{~m}^{-1}[6] \\
a_{N}=-1,2 \times 10^{-26} \mathrm{~m}^{-3}[10] \quad a_{T}=4 \times 10^{-4} \mathrm{~K}^{-1}[6]
\end{gathered}
$$

$n_{0}=3,59[11] T_{0}=297 \mathrm{~K} \lambda_{0}=900,16 \mathrm{~nm}$

$$
N_{0}=100 \mathrm{~m}^{-3}
$$

- la prise en compte de la structure réelle du guide d'onde constituant la cavité optique du laser. Ceci est réalisé par le calcul pour chaque cavité de l'indice effectif $n_{\text {eff }}$ et du facteur de confinement $\Gamma$. Le calcul est effectué de façon bidimensionnelle dans le cas d'une structure à ruban enterré (type «buried ») par la théorie de la « Box » [12] ou guide d'onde rectangulaire,

- le gain de zone active dans chaque cavité est donné par la relation $[13,8]$ :

$$
g_{\mathrm{act}}=\alpha^{\prime}\left[\exp \frac{\Delta E_{\mathrm{F}}-h \nu}{k T}-1\right]
$$

$\alpha^{\prime}$ représente le coefficient de création de paires électron-trou, son expression est déduite des courbes d'absorption obtenues par Henry et al. [14].

$$
\begin{aligned}
& \alpha^{\prime}= 916,184 \exp \frac{E-E_{\mathrm{g} \Gamma}}{1,165 \times 10^{-2}} \text { pour } E \leqslant E_{\mathrm{g} \Gamma} \\
& \alpha^{\prime}=916,184+78618,91\left(E-E_{\mathrm{g} \Gamma}\right)+ \\
& \quad+143602,92\left(E-E_{\mathrm{g} \Gamma}\right)^{2} \text { pour } E \geqslant E_{\mathrm{g} \Gamma}
\end{aligned}
$$

$E_{\mathrm{g} \Gamma}$ représente la largeur de bande interdite pour la vallée $\Gamma$ du GaAlAs, son expression en fonction du taux d'aluminium et de la température est :

$$
\begin{aligned}
& E_{\mathrm{g} \Gamma}=E_{\mathrm{gT}}+1,247 x_{\mathrm{Al}} \text { pour } x_{\mathrm{Al}} \leqslant 0,45 \\
& E_{\mathrm{g} \Gamma}=E_{\mathrm{gT}}+1,247 x_{\mathrm{Al}}+ \\
& +1,147\left(x_{\mathrm{Al}}-0,45\right)^{2} \text { pour } x_{\mathrm{Al}} \geqslant 0,45 \\
& E_{\mathrm{gT}}=1,424+\left(9,6517 \times 10^{-2}-5,405 \times\right. \\
& \left.\times 10^{-4} \frac{T^{2}}{T+204}\right)
\end{aligned}
$$

Enfin, l'analyse sera faite à partir des écarts des pseudo-niveaux de Fermi dans chaque cavité qui représentent les tensions appliquées sur les zones actives. Ils sont reliés aux tensions appliquées et aux courants électriques par les relations :

$$
V_{i}=\frac{1}{q} \Delta E_{\mathrm{F} i}+R_{i} I_{i}
$$

$R_{i}$ représentant la résistance «série » de chaque élément.

\section{Description du modèle.}

3.1 CONDITION D’OSCILlATION. - Le cheminement du rayon lumineux dans la structure est représenté sur la figure 2. Les constantes de propagation complexes $\gamma_{1}$ et $\gamma_{2}$ dans chaque cavité sont données par :

$$
\gamma_{i}=\frac{g_{\text {mod }_{i}}}{2}-j \beta_{i}(i=1,2)
$$




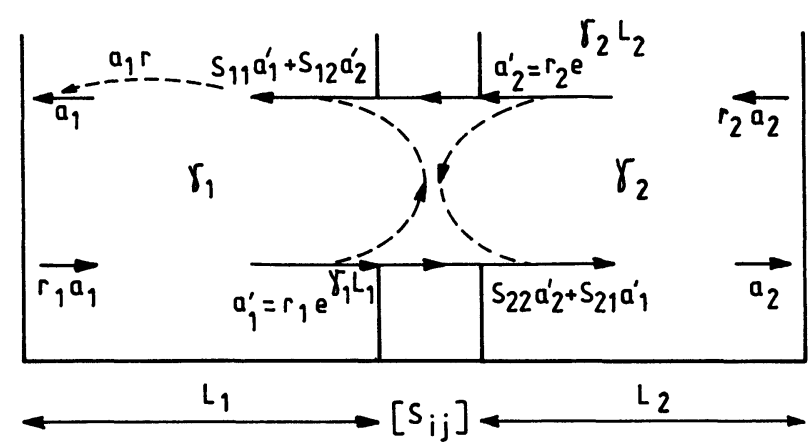

Fig. 2. - Cheminement du rayon : $a_{1}=$ amplitude de départ $a_{1 \mathrm{r}}=$ amplitude après un aller-retour dans la structure.

[The travel of the ray : $a_{1}$ is the amplitude of the starting ray, $a_{1 \mathrm{r}}$ is the amplitude after a round trip.]

avec

$$
\beta_{i}=\frac{2 \pi n_{\mathrm{eff}_{i}}}{\lambda}
$$

et

$$
g_{\bmod _{i}}=\Gamma_{i} g_{\mathrm{act}_{i}}-\alpha_{i}
$$

avec pour chaque cavité :

$g_{\text {mod }_{i}}$ : gain modal de la cavité,

$n_{\text {cff }_{i}} ; \Gamma_{i}$ : indice effectif et facteur de confinement pour le mode considéré,

$\alpha_{i} \quad$ : pertes par diffusion aux interfaces et par absorption par les électrons libres.

L'étude du cheminement du rayon $a_{1}$ dans toute la structure permet d'établir l'expression de l'amplitude $a_{1 \mathrm{r}}$ résultant d'un aller-retour de $a_{1}$ :

$$
a_{1 \mathrm{r}}=\left(Z_{\text {module }}-j Z_{\text {phase }}\right) a_{1}
$$

avec

$$
\begin{aligned}
Z_{\text {modulc }}= & \xi G_{1} G_{2} \cos \left(\varphi_{1}+\varphi_{2}\right)+ \\
& +S_{11} r_{1} G_{1} \cos \varphi_{1}+S_{22} r_{2} G_{2} \cos \varphi_{2} \\
Z_{\text {phasc }}= & \xi G_{1} G_{2} \sin \left(\varphi_{1}+\varphi_{2}\right)+ \\
& +S_{11} r_{1} G_{1} \sin \varphi_{1}+S_{22} r_{2} G_{2} \sin \varphi_{2}
\end{aligned}
$$

avec

$$
\begin{gathered}
\xi=\left(S_{12} S_{21}-S_{11} S_{22}\right) r_{1} r_{2} \\
G_{i}=\exp \left(g_{\bmod _{i}} L_{i}\right) \quad \varphi_{i}=2 \beta_{i} L_{i}
\end{gathered}
$$

La condition d'oscillation de la cavité globale est $a_{1 \mathrm{r}}=a_{1}$. Soit

$Z_{\text {phase }}=0$ équation qui détermine les longueurs d'ondes de résonance notées $\lambda_{r}$ (modes longitudinaux),

$Z_{\text {module }}=1$ équation qui sélectionne le ou les pics lasers et le $\Delta E_{\mathrm{F} 1}$ maximum $\left(\Delta E_{\mathrm{F} 1}\right.$ limite) pour une valeur $\Delta E_{\mathrm{F} 2}$ fixée. C'est la condition d'oscillation sur le gain de la structure.
3.2 PuisSANCE MOdAle SUR LES FACES MiRoIRs. - Pour accéder à l'amplitude de l'émission optique sur chaque miroir, il est nécessaire de prendre en compte la répartition de l'émission spontanée dans chaque mode longitudinal, sur chaque face de la cavité [15], comme représenté sur la figure 3 .

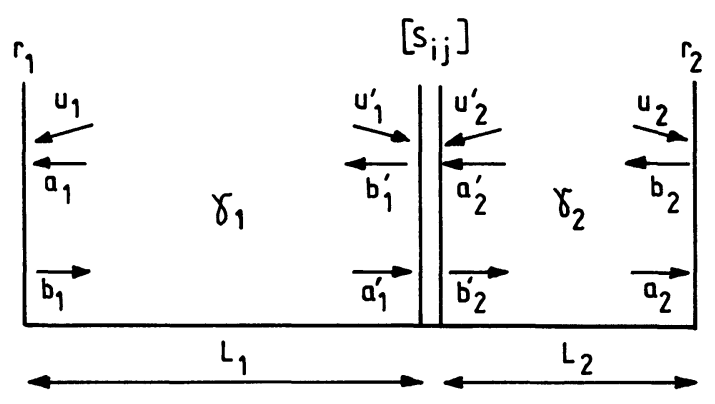

Fig. 3. - Schéma des différents rayons dans la structure avec prise en compte de la répartition de l'émission spontanée (termes $u_{i}$ et $u_{i}^{\prime}$ ).

[Schematic drawing of two sections laser including the equivalents input to the mirror $\left(u_{i}\right)$ and to junction $\left(u_{i}^{\prime}\right)$ of the distributed spontaneous emission.]

La puissance de l'émission spontanée répartie par face et par mode longitudinal est donnée par la relation :

$$
U_{i}=\frac{C_{i}^{\prime}}{2} \frac{G_{i}-1}{L_{i} g_{\bmod _{i}}}
$$

$i=1,2$ indice de cavité

$$
C_{i}^{\prime}=\beta_{i}^{\prime} V h \nu R_{\text {spon }_{i}}
$$

$\beta_{i}^{\prime}=$ coefficient d'émission spontanée

$$
\beta_{i}^{\prime}=\frac{\lambda^{4} \Gamma}{8 \pi^{2}(\Delta \lambda) n_{\text {act }} n_{\text {eff }} n_{g} w d L}
$$

avec

$$
\begin{aligned}
& n_{g}=n_{\text {eff }}-\lambda\left(\mathrm{d} n_{\text {eff }} / \mathrm{d} \lambda\right) \\
& R_{\text {spon }_{i}}=\frac{8 \pi n_{\text {act }_{i}}^{2}}{h^{3} c^{2}} \times \\
& \times \int_{0}^{\infty} \alpha_{(E)}^{\prime} E^{2} \frac{\mathrm{d} E}{\exp \left(\frac{E-\Delta E_{\mathrm{Fi}}}{k T}\right)-1}
\end{aligned}
$$

l'amplitude $u_{i}$ s'en déduit par la relation :

$$
U_{i}=\left|u_{i}\right|^{2} \Delta \nu_{\mathrm{m}}
$$

$\Delta \nu_{\mathrm{m}}$ : espacement entre les modes longitudinaux.

Après avoir exprimé les amplitudes des divers rayons en fonction de $a_{1}$ et de $u_{i}$ et $u_{i}^{\prime}$, l'amplitude du rayon sur le miroir 1 s'exprime alors par la relation :

$$
A_{1}=a_{1}+u_{1}=\frac{d_{1} u_{1}+d_{1}^{\prime} u_{1}^{\prime}+d_{2} u_{2}+d_{2}^{\prime} u_{2}^{\prime}}{1-\left(Z_{\text {module }}-j Z_{\text {phase }}\right)}
$$


avec

$$
\begin{aligned}
& d_{1}=1-S_{22} r_{2} p_{2}^{2} \\
& d_{1}^{\prime}=p_{1}\left[S_{11}\left(1-S_{22} r_{2} p_{2}^{2}\right)+S_{12} S_{21} r_{2} p_{2}^{2}\right] \\
& d_{2}=S_{12} p_{1} p_{2} r_{2} \\
& d_{2}^{\prime}=S_{12} p_{1} \quad p_{i}=\exp \left(\gamma_{i} L_{i}\right)
\end{aligned}
$$

et celle sur le miroir 2 :

$$
A_{2}=a_{2}+u_{2}=\frac{e_{1} u_{1}+e_{1}^{\prime} u_{1}^{\prime}+e_{2} u_{2}+e_{2}^{\prime} u_{2}^{\prime}}{1-\left(Z_{\text {module }}-j Z_{\text {phase }}\right)}
$$

avec

$$
\begin{aligned}
& e_{1}=S_{21} p_{1} p_{2} r_{1} \\
& e_{2}=1-S_{11} p_{1}^{2} r_{1} \\
& e_{1}^{\prime}=S_{21} p_{2} \\
& e_{2}^{\prime}=p_{2}\left[S_{22}\left(1-S_{11} p_{1}^{2} r_{1}\right)+S_{21} S_{12} p_{1}^{2} r_{1}\right]
\end{aligned}
$$

L'intensité optique pour un mode qui s'en déduit est $\Lambda_{1}=A_{1} \cdot A_{1}^{*}$ pour le miroir 1 soit :

$\Lambda_{1}=\frac{\left(\left|d_{1}\right|^{2}+\left|d_{1}^{\prime}\right|^{2}\right)\left|u_{1}\right|^{2}+\left(\left|d_{2}\right|^{2}+\left|d_{2}^{\prime}\right|^{2}\right)\left|u_{2}\right|^{2}}{\left(1-Z_{\text {module }}\right)^{2}+Z_{\text {phasc }}^{2}}$

en considérant que les amplitudes d'émissions spontanées ne sont pas en cohérence de phase entre les deux cavités $\left(u_{i} u_{j}=u_{i}^{\prime} u_{j}=u_{i}^{\prime} u_{j}^{\prime}=0\right)$ mais le sont par contre à l'intérieur de chaque cavité $\left(u_{i}^{\prime}=u_{i}\right)$ [6]. La puissance optique incidente sur un miroir pour un mode longitudinal s'exprime par:

$$
P_{1}=\int_{\Delta \nu_{m}} \Lambda_{1} \mathrm{~d} \nu
$$

pour le miroir 1 qui en supposant que seul $Z_{\text {phase }}$ varie sur le domaine d'intégration $\Delta \nu_{m}$ conduit à :

$P_{1}=\frac{\left(\left|d_{1}\right|^{2}+\left|d_{1}^{\prime}\right|^{2}\right) U_{1}+\left(\left|d_{2}\right|^{2}+\left|d_{2}^{\prime}\right|^{2}\right) U_{2}}{1-Z_{\text {module }}^{2}}$

de même sur le miroir 2 :

$$
P_{2}=\frac{\left(\left|e_{1}\right|^{2}+\left|e_{1}^{\prime}\right|^{2}\right) U_{1}+\left(\left|e_{2}\right|^{2}+\left|e_{2}^{\prime}\right|^{2}\right) U_{2}}{1-Z_{\text {module }}^{2}}
$$

3.3 EXPRESSION DES COURANTS ÉLECTRIQUES DANS CHAQUE CAVITÉ. - La densité de photons $S_{i}$, par mode, dans la zone active de chaque cavité s'exprime en fonction de la puissance moyenne optique $\left\langle P_{i}\right\rangle$ dans chaque cavité $i$.

$$
\begin{gathered}
\left\langle P_{i}\right\rangle=\frac{1}{L_{i}} \int_{0}^{L_{i}}\left(P_{i} \mathrm{e}^{\left.-g_{\bmod _{i} z} z P_{i}^{\prime} \mathrm{e}^{g_{\bmod _{i}}\left(z-L_{i}\right)}\right) \mathrm{d} z}\right. \\
S_{i}=\frac{\left\langle P_{i}\right\rangle \Gamma_{i}}{\frac{c}{n_{\mathrm{eff}_{i}} h \nu w_{i} d_{i}}}
\end{gathered}
$$

Le courant électrique traversant chaque cavité est régi par l'équation de continuité :

$$
\frac{\mathrm{d} N}{\mathrm{~d} t}=\frac{I}{q d w L}-R_{\mathrm{spon}}-\frac{c}{n_{\mathrm{eff}}} \sum_{j}\left(g_{\mathrm{act}}^{j} S^{j}\right)
$$

$j$ : indice des modes longitudinaux.

Ce qui en régime permanent conduit aux équations :

$$
\begin{aligned}
& I_{1}=q d_{1} w_{1} L_{1}\left[R_{\mathrm{spon}_{1}}+\frac{c}{n_{\mathrm{eff}_{1}}} \sum_{j}\left(g_{\mathrm{act}_{1}}^{j} S_{1}^{j}\right)\right] \\
& I_{2}=q d_{2} w_{2} L_{2}\left[R_{\mathrm{spon}_{2}}+\frac{c}{n_{\mathrm{eff}_{2}}} \sum_{j}\left(g_{\mathrm{act}_{2}}^{j} S_{2}^{j}\right)\right]
\end{aligned}
$$

\section{Résultats.}

Ce paragraphe présente et exploite les différentes courbes calculées, caractéristiques du mode de fonctionnement du couplage électro-optique des deux cavités.

La présentation des résultats en fonction des $\Delta E_{\mathrm{F} 1}$ et $\Delta E_{\mathrm{F} 2}$ apporte un éclairage supplémentaire par rapport aux résultats en fonction de $I_{1}$ et $I_{2}[4,16,17,3]$, jusqu'ici présentés.

Ce modèle permet d'obtenir toutes les courbes caractéristiques du fonctionnement des structures lasers à cavités couplées :

- évolution de l'écart des pseudo-niveaux de Fermi en fonction des courants,

- caractéristiques puissance lumineuse-courants, - spectres $P(\lambda)$ en puissance et en intensité $L(\lambda)$

- évolution du seuil laser de la structure en fonction des conditions de polarisation.

Il permet, par ailleurs, d'évaluer la sélectivité spectrale de telles structures, de mettre en évidence et d'expliquer :

- les trois zones de fonctionnement de ces structures,

- l'évolution de la longueur d'onde laser en fonction des conditions de polarisation.

Afin d'illustrer le fonctionnement de ces structures, deux types de lasers à cavités couplées ont été simulés :

- une structure de type «long long» avec une forte interaction optique, $\mathrm{C}^{3}$.

- une structure de type «long court» de type

Le tableau I reporte la valeur des paramètres utilisés dans l'analyse numérique et les dimensions géométriques des cavités pour les deux types de structures étudiées.

La densité de porteurs injectés dans la zone active d'une cavité est reportée sur la figure 4 en fonction de l'écart des pseudo-niveaux de Fermi. Sur la figure 5 sont tracées les variations des indices de 
Tableau I. - Valeur des paramètres utilisés dans la simulation numérique.

[Values of the parameters used in the numerical simulation.]

$i \quad$ : indice de cavité $i=1,2$

$d_{i} \quad$ : épaisseur de zone active $d_{1}=d_{2}=0,1 \mu \mathrm{m}$

$w_{i} \quad$ largeur du ruban de zone active $w_{1}=$ $w_{2}=10 \mu \mathrm{m}$

$L_{i} \quad$ : longueur de la cavité

$T \quad$ : température $T=293 \mathrm{~K}$

$x_{\mathrm{Ali}}$ : concentration d'aluminium dans la zone active $x_{\mathrm{A} 11}=x_{\mathrm{A} 12}=0$

$r_{i} \quad$ : coefficient de réflexion en amplitude des miroirs $r_{1}=r_{2}=0,5916$

$\alpha_{i} \quad$ : pertes optiques latérales de cavité $\alpha_{1}=\alpha_{2}=20 \mathrm{~cm}^{-1}$

$N_{\mathrm{A} i} \quad$ : dopage de zone active $N_{\mathrm{A} 1}=N_{\mathrm{A} 2}=$ $10^{17} \mathrm{~cm}^{-3}$

$n_{j}=3,4$ : indice dans les zones adjacentes au ruban de zone active

$\alpha_{j}=1000 \mathrm{~cm}^{-1}$ absorption dans les zones adjacentes au ruban de zone active

Structure long-long :

$$
\begin{aligned}
L_{1} & =240 \mu \mathrm{m} & L_{2} & =180 \mu \mathrm{m} \\
S_{11} & =S_{22}=0,005 & S_{12} & =S_{21}=0,8
\end{aligned}
$$

Structure long-court :

$$
\begin{aligned}
L_{1} & =170 \mu \mathrm{m} & L_{2} & =40 \mu \mathrm{m} \\
S_{11} & =S_{22}=0,5 & S_{12} & =S_{21}=0,68
\end{aligned}
$$

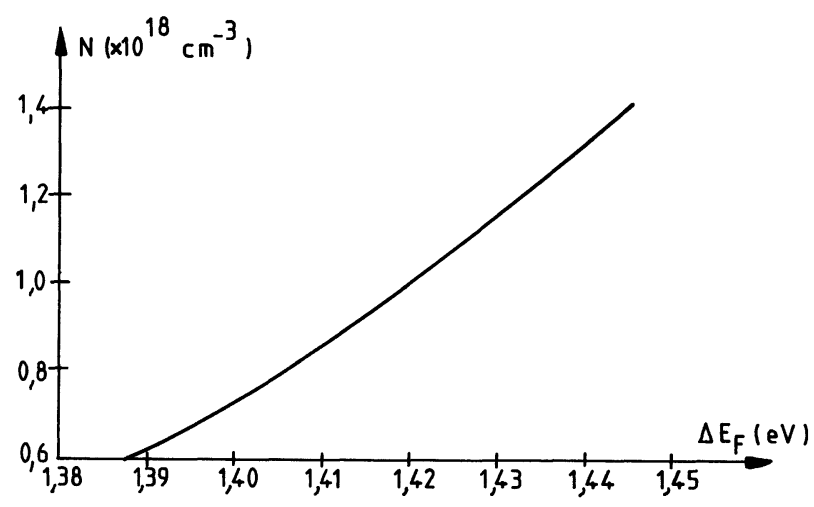

Fig. 4. - Densité de porteur injectée dans la zone active en fonction de l'écart des pseudo-niveaux de Fermi.

[Injected carriers density in the active zone as function of the quasi Fermi levels separation.]

zone active $n_{\text {act }}$ et effectif $n_{\text {eff }}$ dans une cavité en fonction de $\Delta E_{\mathrm{F}}$ pour une longueur d'onde fixée.

4.1 CARACTÉRISTIQUES $\Delta E_{\mathrm{F} 1}\left(I_{1}\right)_{\Delta E_{\mathrm{F} 2} \cdot}$ - Ces caractéristiques reportées sur les figures 6 et 7 présentent la même allure pour les deux types de structures

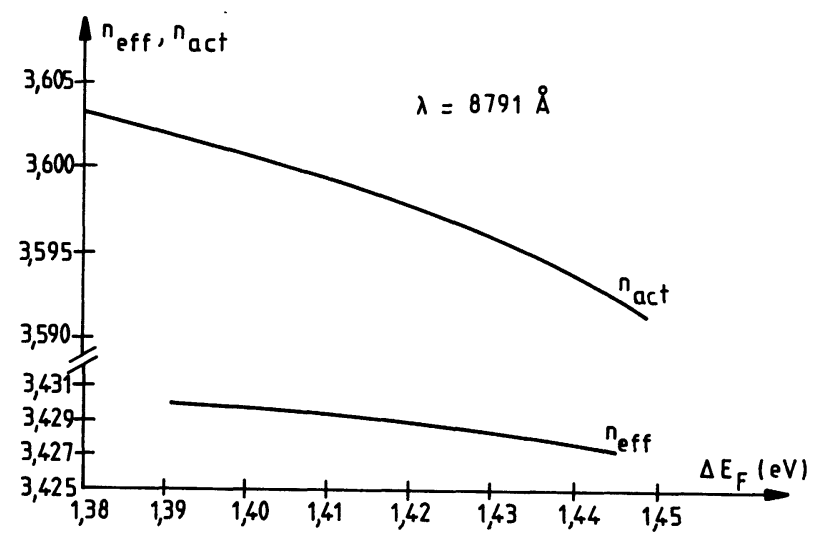

Fig. 5. - Variations de l'indice de zone active $n_{\text {act }}$ et de l'indice effectif $n_{\text {eff }}$ en fonction de l'écart des pseudoniveaux de Fermi.

[Active zone index $n_{\text {act }}$ and effective index $n_{\text {eff }}$ variations versus quasi Fermi levels separation.]

étudiées. Leur analyse permet d'expliquer le comportement des lasers à cavités couplées et notamment les raisons de la variation de la longueur d'onde d'émission.

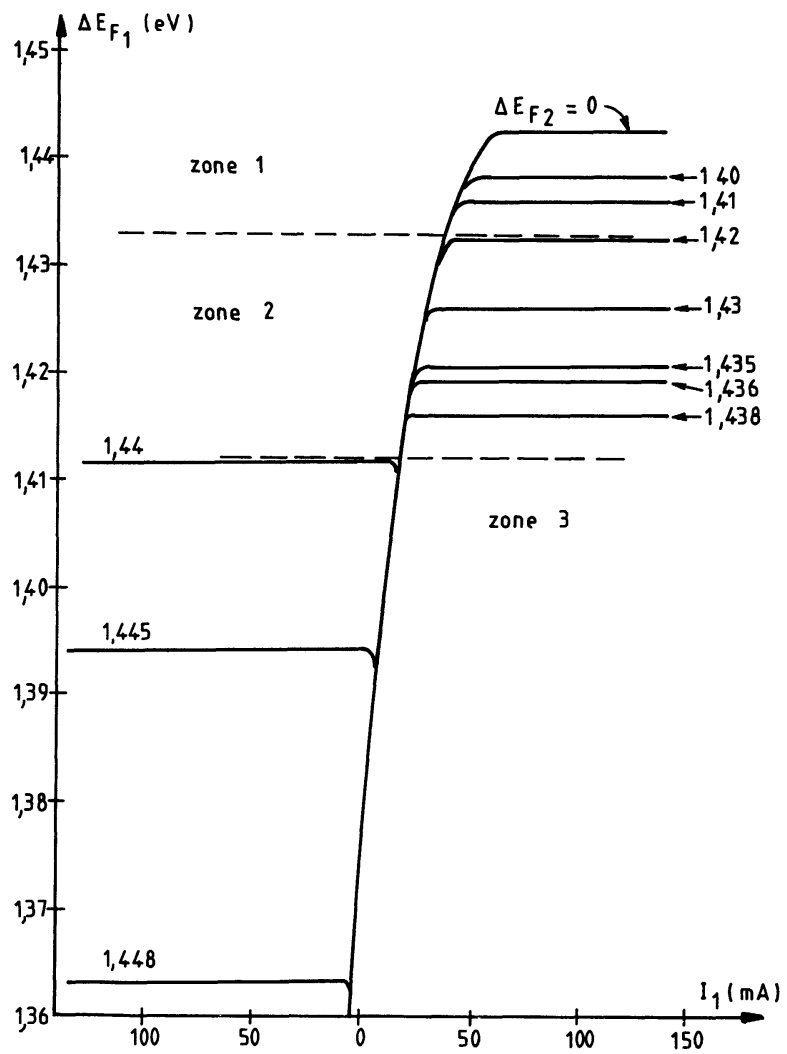

Fig. 6. - Evolution de l'écart des pseudo-niveaux de Fermi de la cavité 1 en fonction du courant traversant cette cavité pour $\Delta E_{\mathrm{F} 2}$ fixé. Cas de la structure long-long.

[Evolution of the quasi Fermi levels separation as function of the current $I_{1}$ for cavity one at Const. $\Delta E_{\mathrm{F} 2}$. Case of the long-long structure.] 


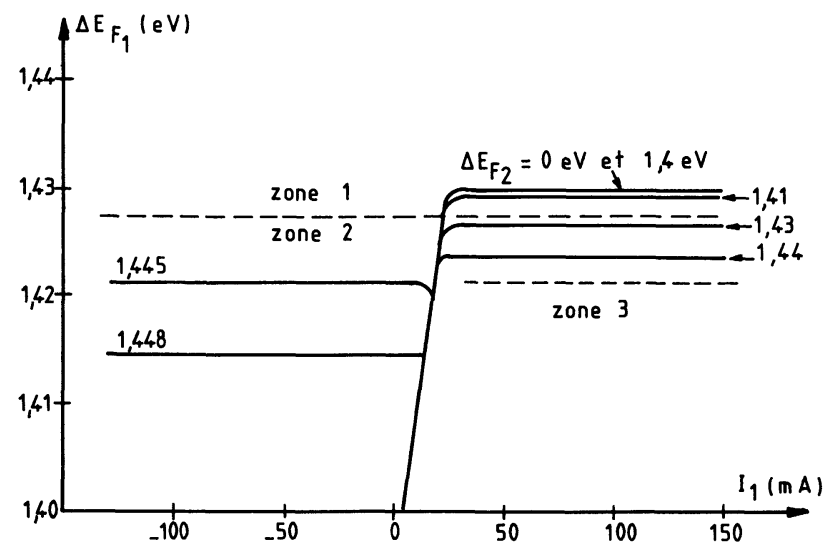

Fig. 7. - Evolution de l'écart des pseudo-niveaux de Fermi de la cavité 1 en fonction du courant traversant cette cavité pour $\Delta E_{\mathrm{F} 2}$ fixé. Cas de la structure long-court.

[Evolution of the quasi Fermi levels separation as function of the current $I_{1}$ for cavity one, at Const. $\Delta E_{\mathrm{F} 2}$. Case of the short-long structure.]

Pour chaque valeur de $\Delta E_{\mathrm{F} 2}$ appliquée, ces courbes présentent un palier de saturation de $\Delta E_{\mathrm{F} 1}$ lorsque $I_{1}$ croît indéfiniment. Cette limite de $\Delta E_{\mathrm{F} 1}$ est pratiquement atteinte dès que $I_{1}$ est supérieur à $I_{\text {th }}\left(I_{\text {th }}\right.$ étant le seuil de l'ensemble de deux cavités). Ceci se traduit par une quasi-constance du gain, de la densité de porteurs et de l'indice effectif de la cavité 1 en fonction des très faibles variations de $\Delta E_{\mathrm{F} 1}$, dès que $I_{1}$ dépasse $I_{\mathrm{th}}$. Ce phénomène a été couramment pris comme hypothèse de travail par de nombreux auteurs.

Il en résulte que la longueur d'onde émise reste constante à $\Delta E_{\mathrm{F} 2}$ fixée quelles que soient les valeurs de $I_{1}$ supérieures au seuil $I_{\text {th }}$. On remarque, par ailleurs, que la valeur de $\Delta E_{\mathrm{F} 1 \text { lime }}$ décrôit avec $\Delta E_{\mathrm{F} 2}$ et qu'en fonctionnement laser, la longueur d'onde émise varie par palier avec $\Delta E_{\mathrm{F} 2}$ (Fig. 8).

Selon le signe de $g_{\text {act }_{i}^{j}}^{j}\left(i \mathrm{n}^{\circ}\right.$ de cavité, $j \mathrm{n}^{\circ}$ de mode $)$

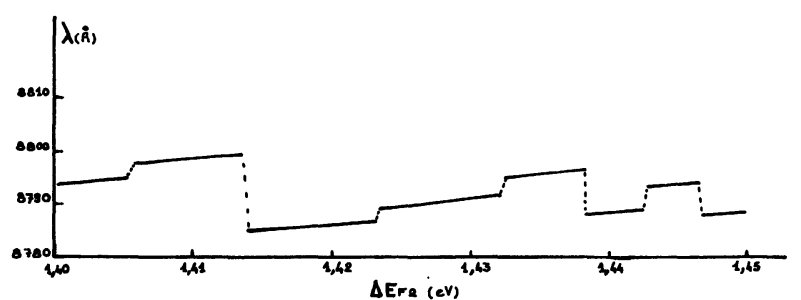

Fig. 8. - Evolution du pic laser $\lambda_{\text {lim }}$ en fonction de $\Delta E_{\mathrm{F} 2}$ dans le cas de la structure long-long.

[Emitted peack $\lambda_{\text {lim }}$ shift as function of $\Delta E_{\mathrm{F} 2}$. Case of the long-long structure.]

qui indique si la cavité est ou non stimulée pour le mode laser $j$ émis, on peut définir trois zones dans le plan $\Delta E_{\mathrm{F} 1}\left(I_{1}\right)_{\Delta E_{\mathrm{F}}}$.

Le tableau II résume le comportement de la structure dans chaque zone. La description du fonctionnement lorsque $\Delta E_{\mathrm{F} 2}$ croît à partir de 0 peut se faire comme suit :

- La zone I ou la cavité 1 est stimulée $\left(g_{\mathrm{act}}^{j}>0\right)$ et la cavité 2 absorbante $\left(g_{\text {act }}^{j}<0\right)$ pour le mode laser $j$ émis. Dans cette zone, lorsque la cavité couplée est au-dessus du seuil, (à ce moment $\Delta E_{\mathrm{F} 1}$ a atteint sa valeur de saturation pour la valeur de $\Delta E_{\mathrm{F} 2}$ appliquée) si on fait croître $I_{1}$, la densité de photons $S_{1}$ émise dans la cavité 1 dans le mode laser $j$ croît et la cavité 2 reçoit plus de lumière. D'après la relation (25) $I_{2}$ décroît. On a donc $s=\mathrm{d} I_{2} / \mathrm{d} I_{1}<0$ avec $I_{1}$ positif croissant et $I_{2}$ décroissant jusqu'à $(-\infty)$. Lorsque $\Delta E_{\mathrm{F} 2}$ augmente, la cavité 2 devient de moins en moins absorbante et la cavité 1 de moins en moins stimulée. Donc dans cette zone $s$ croît avec $\Delta E_{\mathrm{F} 2}$. La limite de cette zone correspond à une valeur $\Delta E_{\mathrm{F}_{2}}$ telle que pour cette valeur $g_{\text {act }_{2}}=0$ soit $\Delta E_{\mathrm{F}_{1}}=h \nu_{j}$ et $s=0$ (transparence de la cavité 2 à la lumière provenant de la cavité 1 ).

Tableau II. - Synthèse du comportement d'un laser à cavités couplées.

[A synthesis of coupled cavity laser behaviour.]

A $\Delta E_{\mathrm{F} 2}$ donné lorsque $\Delta E_{\mathrm{F} 1}$ augmente de $0 \rightarrow \Delta E_{\mathrm{F} 1 \text { lim }}$ $g_{\text {act } 1}$ croît $\rightarrow g_{\text {act } 1 \text { lim }}$

$P_{1}, P_{2},\langle P\rangle, S \rightarrow+\infty \quad \lambda_{\text {pic }} \rightarrow \lambda_{\text {lim }}$

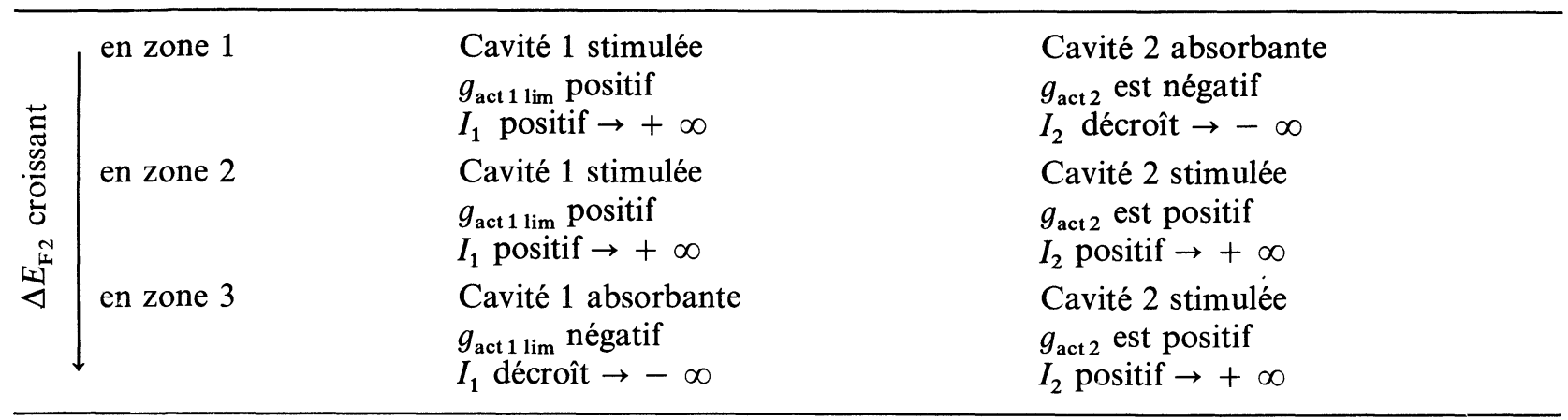


- La zone II où $\Delta E_{\mathrm{F} 2}$ est supérieur à $\Delta E_{\mathrm{F}_{1}}$ et où les deux cavités sont stimulées : $g_{\text {act }_{1}}^{j}>0$ et $g_{\text {act }_{2}}^{j}>0$. Les deux courants sont positifs et croissants $s=\mathrm{d} I_{2} / \mathrm{d} I_{1}>0$.

Dans cette zone, au fur et à mesure que $\Delta E_{\mathrm{F} 2}$ croît, $g_{\text {act }_{2}}$ croît en valeur positive; $g_{\text {act }_{1}}$ décroît en restant également positif. La cavité 2 devient de plus en plus stimulée, la cavité 1 de moins en moins. L'apport du gain de chaque cavité pour vaincre les pertes globales de la structure va ainsi se modifier au profit de la cavité 2 . C'est donc en fait dans cette zone et lorsque $s$ passe par son maximum que s'effectue le «retournement» de la structure en ce qui concerne l'influence de chaque cavité sur le fonctionnement du laser à cavités couplées.

La limite de cette seconde zone sera atteinte lorsque $g_{\text {act }_{1}}^{j}=0$ soit pour $\Delta E_{\mathrm{F} 2}$ tel que $\Delta E_{\mathrm{F} 1 \text { lim }}=h \nu_{j}$. La cavité 1 est alors transparente pour la lumière provenant de la cavité 2 et nous avons $s=0$.

La zone III enfin où les valeurs limites de $\Delta E_{\mathrm{F} 1}$ correspondant aux $\Delta E_{\mathrm{F} 2}$ appliqués rendent $g_{\text {act }_{1}}^{j}<0$. Les rôles des deux cavités sont intervertis par rapport à la zone $\mathrm{I}$; c'est la cavité 2 qui pilote la structure. Ici $s=\mathrm{d} I_{2} / \mathrm{d} I_{1}<0$ mais c'est $I_{1}$ qui diminue pendant que $I_{2}$ croît, $s$ décroît.

4.2 Courbes De PUissance $P_{1}\left(I_{1}\right)_{\Delta E_{\mathrm{F} 2}}$. - Les courbes $P_{1}\left(I_{1}\right)_{\Delta E_{\mathrm{F} 2}}$ correspondant aux valeurs de $\Delta E_{\mathrm{F} 2}$ traitées dans le paragraphe précédent ont été calculées, figures 9 et 10 . Ces courbes permettent de déterminer le seuil global de la structure et le rendement différentiel. Elles permettent également d'expliquer le comportement des structures lasers à cavités couplées en fonction des courants injectés, présentation qui a été utilisée dans les publications de résultats expérimentaux antérieurs.

4.2.1 Etude du seuil. - On constate que le seuil est d'autant plus faible que $\Delta E_{\mathrm{F} 2}$ est élevé.

4.2.2 Rendement quantique différentiel $\eta_{1 \mathrm{~d}}=$ $\mathrm{d} P_{1} / \mathrm{d} I_{1}$. - Les valeurs de $\eta_{1 \mathrm{~d}}$ paramétrées en $\Delta E_{\mathrm{F} 2}$ sont reportées dans le tableau III. Dans les zones I et II $\eta_{1 \mathrm{~d}}$ croît avec $\Delta E_{\mathrm{F} 2}$. A la limite de la zone II, on observe un comportement « quasi $Q$ switched " du laser couplé : à une très faible variation de $I_{1}$ correspond une variation considérable de $P_{1}$.

4.2.3 Analyse du comportement de la structure à courant $I_{1}$ constant. - Les informations à ce sujet sont données par les intersections successives avec les courbes $P_{1}\left(I_{1}\right)$ d'une verticale tracée dans ce plan. Il est utile de rappeler (cf. paragraphe 4.1) que chacune de ces courbes correspond à un seul mode laser (lorsque le spectre est monomode) (cf. paragraphe 5); de sorte qu'une étude à $I_{1}$ constant et

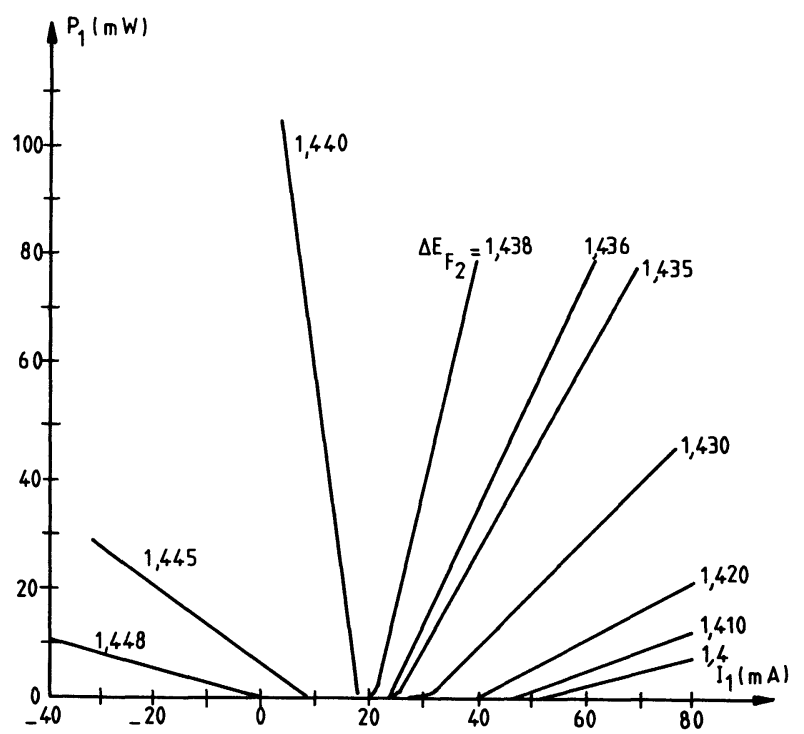

Fig. 9. - Caractéristiques puissance lumineuse-courant de la cavité 1 en fonction de l'écart des pseudo-niveaux de Fermi dans la cavité 2 ; pour la structure long-long.

[Light versus current $I_{1}$ characteristics in cavity 1 at various values of $\Delta E_{\mathrm{F} 2}$. Case of the long-long structure.]

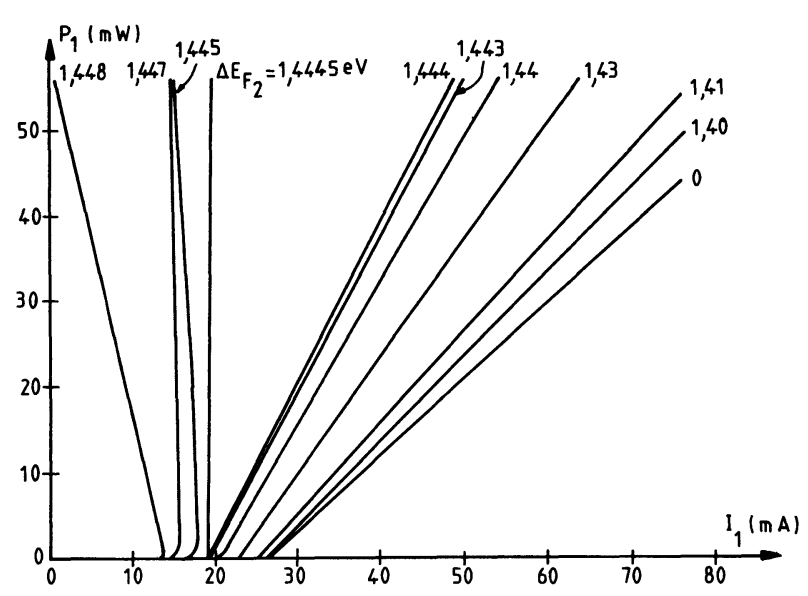

Fig. 10. - Caractéristiques puissance lumineuse-courant de la cavité 1 en fonction de l'écart des pseudo-niveaux de Fermi dans la cavité 2 ; pour la structure long-court.

[Light versus current $I_{1}$ characteristic at various values of $\Delta E_{\mathrm{F} 2}$. Case of short-long structure.]

$I_{2}$ variable conduit à des variations simultanées de $\Delta E_{\mathrm{F} 1}$ et $\Delta E_{\mathrm{F} 2}$. Contrairement à l'explication suggérée par W. T. Tsang, N. A. Olson, R. A. Logan [16], $n_{\text {eff }_{1}}$ varie, bien que $I_{1}$ soit maintenu constant. L'évolution du pic laser ne peut pas être prévue de façon simple par le défilement des coïncidences entre les modes invariants de la cavité 1 et ceux de la cavité 2 . Les sauts de modes lorsque $I_{2}$ varie sont dus aux variations simultanées de $n_{\mathrm{eff}_{1}}, n_{\mathrm{eff}_{2}}, g_{\text {mod }_{2}}$ et $g_{\text {mod }_{2}}$, ce qui se traduit par des sauts de modes irréguliers, tels que l'illustre la figure 13. 
Tableau III. - Valeurs de $\lambda_{\text {lim }}, I_{1 \text { seuil }}$ et $\eta_{1 \mathrm{~d}}$ en fonction de $\Delta E_{\mathrm{F} 2}$.

$\left[\lambda_{\text {lim }}, I_{1 \mathrm{th}}\right.$ and $\eta_{1 \mathrm{~b}}$ values as function of $\Delta E_{\mathrm{F} 2}$.]

Structure long-long

\begin{tabular}{|c|c|c|c|c|c|c|c|c|c|}
\hline$\Delta E_{\mathrm{F} 2} \mathrm{eV}$ & 1,4 & 1,41 & 1,42 & 1,43 & 1,435 & 1,438 & 1,44 & 1,445 & 1,448 \\
\hline$\lambda_{\lim } \AA$ & 8793,88 & 8798,58 & 8785,99 & 8790,89 & 8795,936 & 8796,05 & 8788,767 & 8794,375 & 8788,036 \\
\hline$I_{1 \text { seuil }} \mathrm{mA}$ & 51 & 46 & 40 & 31 & 25,5 & 21 & 18 & 8,5 & 0 \\
\hline$\eta_{1 \mathrm{D}}$ & 0,28 & 0,38 & 0,54 & 1,04 & 1,8 & 4,3 & $-7,3$ & $-0,72$ & $-0,26$ \\
\hline
\end{tabular}

Structure long-court

\begin{tabular}{|c|c|c|c|c|c|c|c|c|c|c|}
\hline$\Delta E_{\mathrm{F} 2} \mathrm{eV}$ & 0 & 1,4 & 1,41 & 1,43 & 1,44 & 1,444 & 1.44 & 1,445 & 1,447 & 1,448 \\
\hline$\lambda_{\lim } \AA$ & 8772,32 & 8766,35 & 8766,34 & 8790,95 & 8815,79 & 8815,8 & 8736,97 & 8736,99 & 8761,92 & 8762,67 \\
\hline$I_{1 \text { seuil }} \mathrm{mA}$ & 26,6 & 26,3 & 25 & 23 & 20,6 & 19 & 19 & 18 & 16 & 14 \\
\hline$\eta_{1 \mathrm{D}} \frac{\mathrm{mW}}{\mathrm{mA}}$ & 0,9 & 0,98 & 1,06 & 1,33 & 1,66 & 1,84 & 53 & -32 & $-53,8$ & $-3,6$ \\
\hline
\end{tabular}

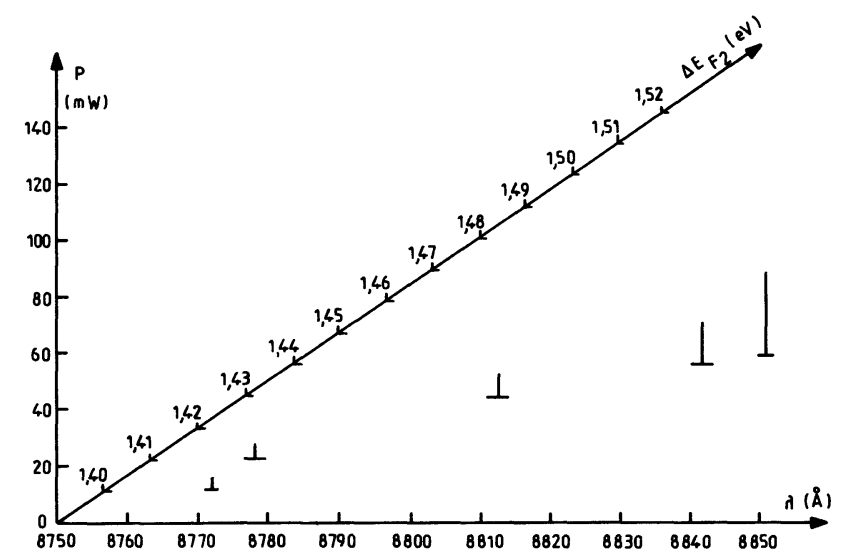

Fig. 11. - Evolution du spectre d'émission en fonction de $\Delta E_{\mathrm{F} 2}$ pour un courant $I_{1}$ constant $I_{1}=30 \mathrm{~mA}$ pour la structure long-court.

[Evolution of the emitted spectrum as function of $\Delta E_{\mathrm{F} 2}$ at current level $I_{1}=30 \mathrm{~mA}$. Case of short-long structure.]

La figure 11 représente les longueurs d'ondes émises à $I_{1}=30 \mathrm{~mA}$ pour différents $\Delta E_{\mathrm{F} 2}$. Déduite des courbes $P_{1}\left(I_{1}\right)$, elle illustre bien les sauts de modes observés expérimentalement. De plus, on y constate que la puissance émise d'un mode à l'autre croît lorsque $\Delta E_{\mathrm{F} 2}$ augmente.

\section{Spectre d'émission.}

Conformément aux prévisions de Marcuse et T. P. Lee [18], les longueurs d'onde résonantes sont irrégulièrement espacées (Fig. 13).
Les figures 12 et 13 présentent les spectres d'émission obtenus aux points de fonctionnement indiqués dans les cas de cavités longue-courte de type $\mathrm{C}^{3}$ et longue-longue à fort couplage.

Ces spectres sont sensibles aux éléments de la matrice « $S$ », aux longueurs respectives des cavités. Alors qu'un fonctionnement monomode est obtenu pratiquement dès que le seuil laser de la cavité couplée est franchi dans le premier cas, dans le second cas, ce mode de fonctionnement ne se produit qu'à niveau de puissance élevé $P \approx 10 \mathrm{~mW}$. Ces résultats concordent avec ceux obtenus expérimentalement par T. P. Lee et al. [19]. Les sauts de mode se produisent surtout lorsque la cavité modulatrice est soit absorbante, soit légèrement stimulée.

La sélectivité du système est chiffrée par le rapport $\delta=\left(P_{1} / \Sigma P_{1 j}\right)$ de la puissance émise dans le mode dominant à la puissance totale émise. Ce rapport est donné dans le tableau IV. Les figures 8 et 11 illustrent l'évolution du pic laser en fonction des conditions de polarisation. Dans certains cas, il peut apparaître simultanément deux pics lasers pour les valeurs de $\Delta E_{\mathrm{F} 2}$ correspondant à des changements de paliers sur la figure 6 ; un exemple en est porté sur la figure 12 .

\section{Largeur de raie.}

L'expression des intensités donnée dans la relation (18) permet de tracer les spectres en intensité et d'en déduire les largeurs de raies théoriques. La figure 14 donne les largeurs de raies à divers niveaux de puissance. Les largeur's à mi-hauteur obtenues pour ces pics sont les suivantes : 


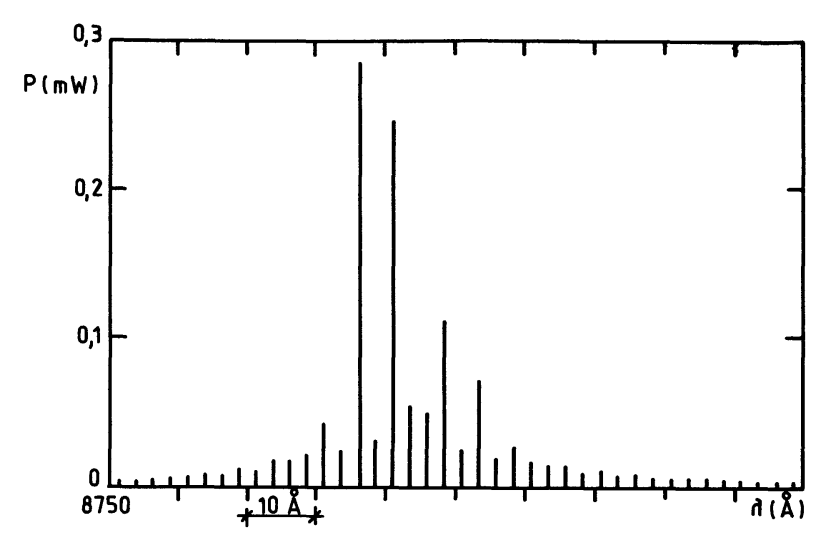

a)

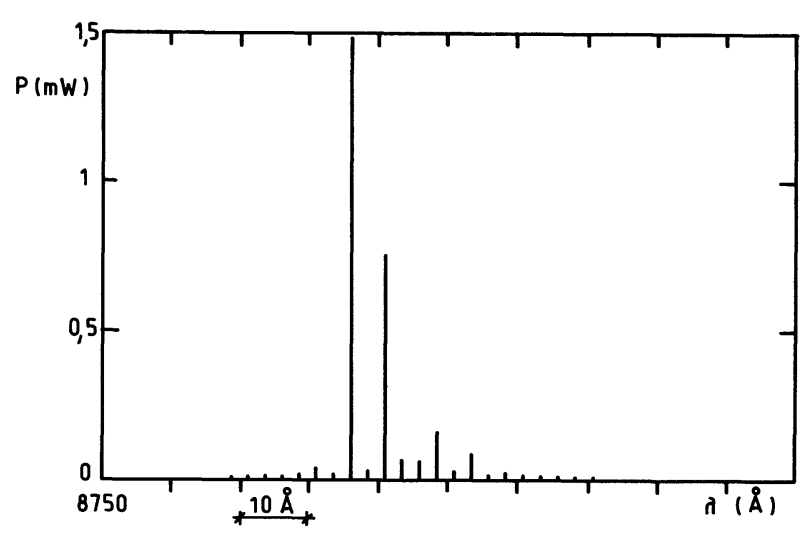

b)

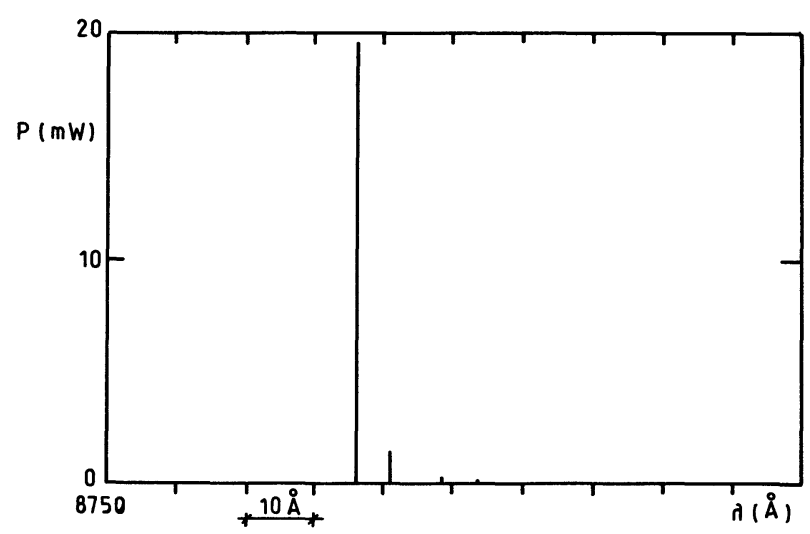

c)

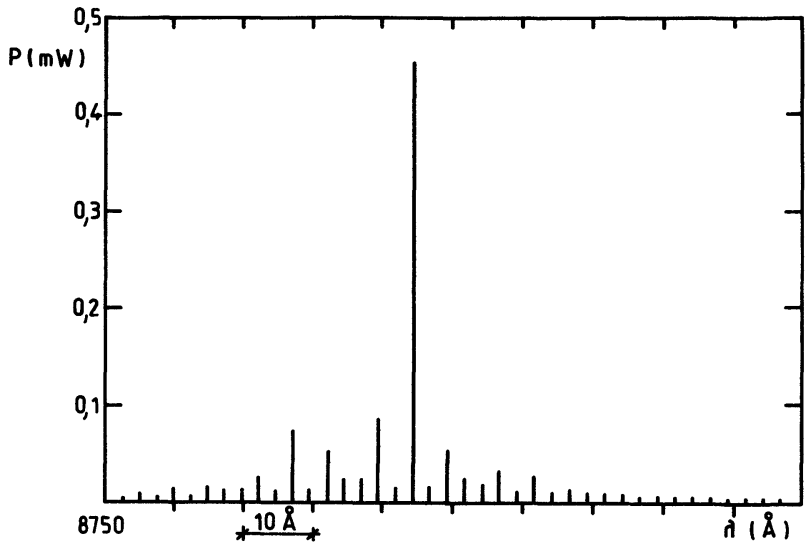

(d)

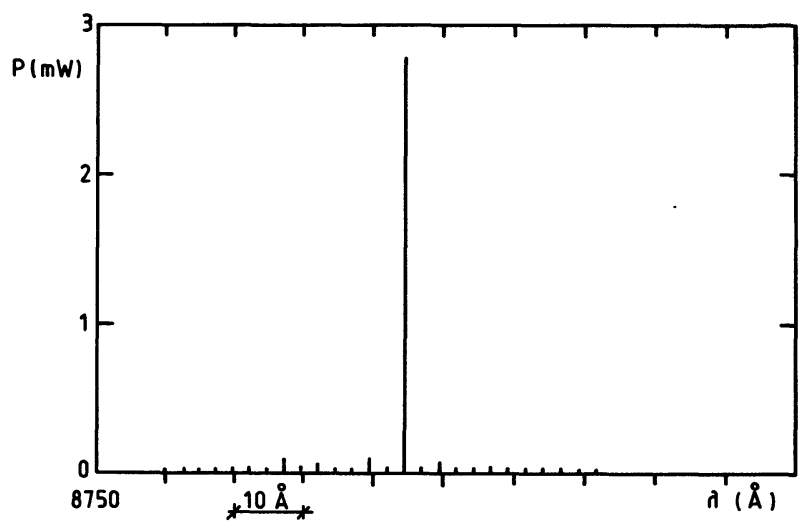

(')

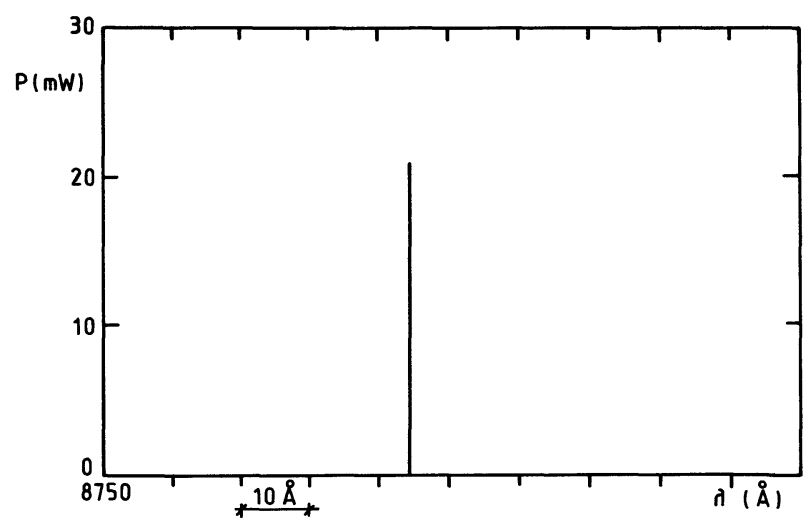

f)

Fig. 12. - Spectres d'émissions de la structure long-long.

\begin{tabular}{cccc} 
& $\Delta E_{\mathrm{F} 2}(\mathrm{eV})$ & $I_{1}(\mathrm{~mA})$ & $I_{2}(\mathrm{~mA})$ \\
\cline { 2 - 4 } $\mathrm{a}$ & 1,42 & 42 & 19 \\
$\mathrm{~b}$ & 1,42 & 45,4 & 19,6 \\
$\mathrm{c}$ & 1,42 & 79,5 & 26,4 \\
$\mathrm{~d}$ & 1,445 & 6,85 & 56,7 \\
$\mathrm{e}$ & 1,445 & 3,55 & 69,2 \\
$\mathrm{f}$ & 1,445 & -22 & 165,5
\end{tabular}

[Calculated emitted spectra. Case of the long-long structure.] 

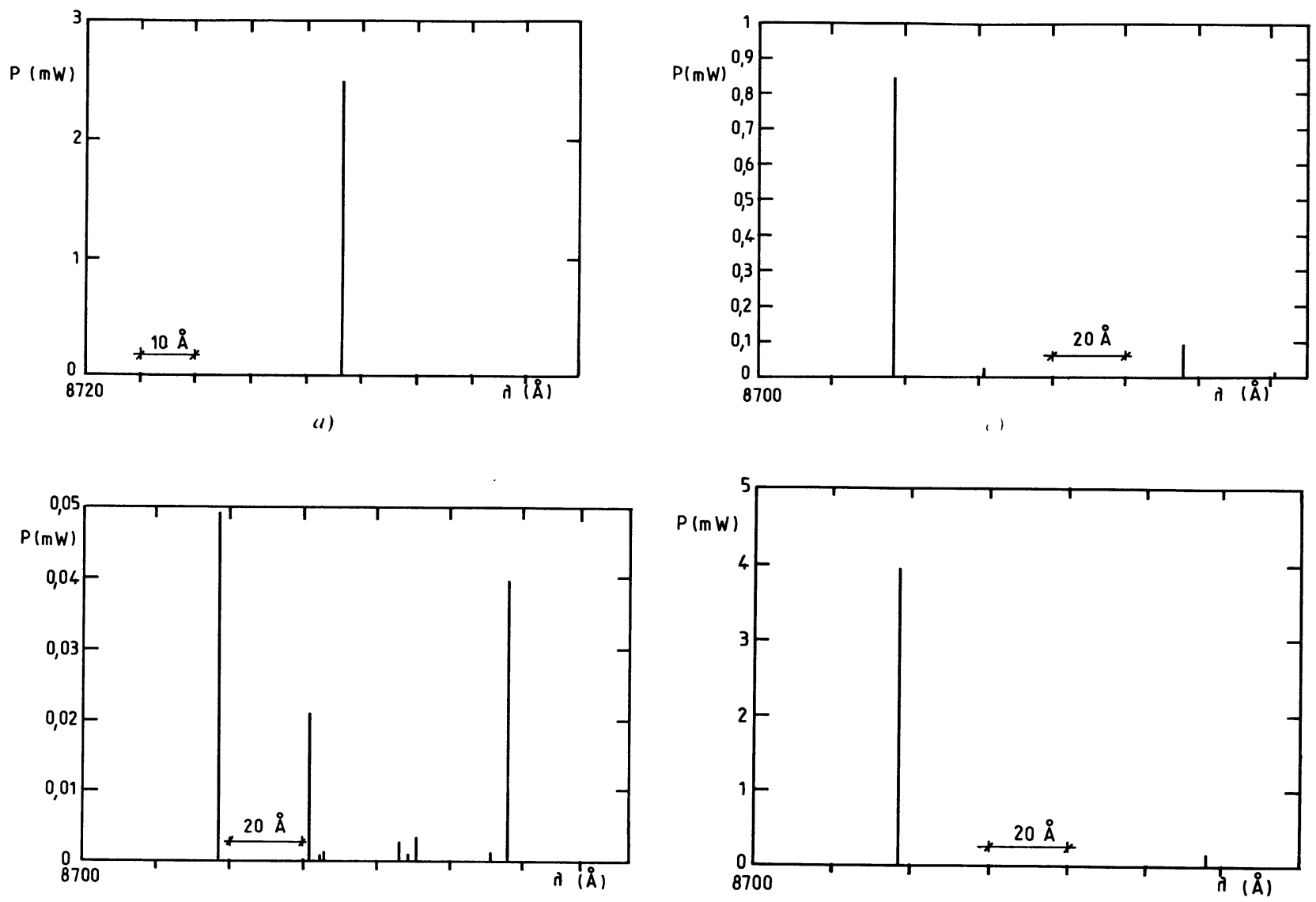

h

d)

Fig. 13. - Spectres d'émissions de la structure long-court :

\begin{tabular}{llll} 
& $\Delta E_{\mathrm{F} 2}(\mathrm{eV})$ & $I_{1}(\mathrm{~mA})$ & $I_{2}(\mathrm{~mA})$ \\
\cline { 2 - 4 } $\mathrm{a}$ & 1,41 & 28 & 2,65 \\
$\mathrm{~b}$ & 1,4445 & 18,8 & 11 \\
$\mathrm{c}$ & 1,4445 & 19 & 12,5 \\
$\mathrm{~d}$ & 1,4445 & 19,1 & 18,1
\end{tabular}

[Calculated emitted spectra. Case of the short-long structure.]

Tableau IV. - Coefficient de sélectivité $\delta$ en fonction de la puissance émise et de $\Delta E_{\mathrm{F} 2}$.

[Rate of selectivity, $\delta$, as function of the emitted power and of $\Delta E_{\mathrm{F} 2}$.]

Structure long-long

\begin{tabular}{|l|ll|lc|cc|ccc|ccc|}
\hline$\Delta E_{\mathrm{F} 2}(\mathrm{eV})$ & \multicolumn{2}{|c|}{1,435} & \multicolumn{2}{|c|}{1,438} & \multicolumn{2}{|c|}{1,44} & \multicolumn{3}{|c|}{1,445} & \multicolumn{3}{|c|}{1,448} \\
\hline$P_{1}(\mathrm{~mW})$ & 11 & 17 & 8 & 18 & 2 & 30 & 1 & 4 & 26 & 1 & 4 & 8 \\
\hline$\delta$ & 0,928 & 0,953 & 0,822 & 0,915 & 0,392 & 0,962 & 0,371 & 0,74 & 0,968 & 0,34 & 0,805 & 0,886 \\
\hline
\end{tabular}

Structure long-court

\begin{tabular}{|l|ll|lll|lll|l|l|l|l|l|}
\hline$\Delta E_{\mathrm{F} 2}(\mathrm{eV})$ & 0 & & 1,4 & & 1,41 & 1,43 & 1,44 & 1,4445 & 1,445 & 1,447 & 1,448 \\
\hline$P_{1}(\mathrm{~mW})$ & 4 & 47 & 0,4 & 46 & 3 & 40 & 8 & 5 & 8 & 5 & 5 & 4 \\
\hline$\delta$ & 0,9897 & 0,9994 & 0,9165 & 0,999 & 0,981 & 0,9987 & 0,996 & 0,995 & 0,9817 & 0,989 & 0,999 & 0,995 \\
\hline
\end{tabular}



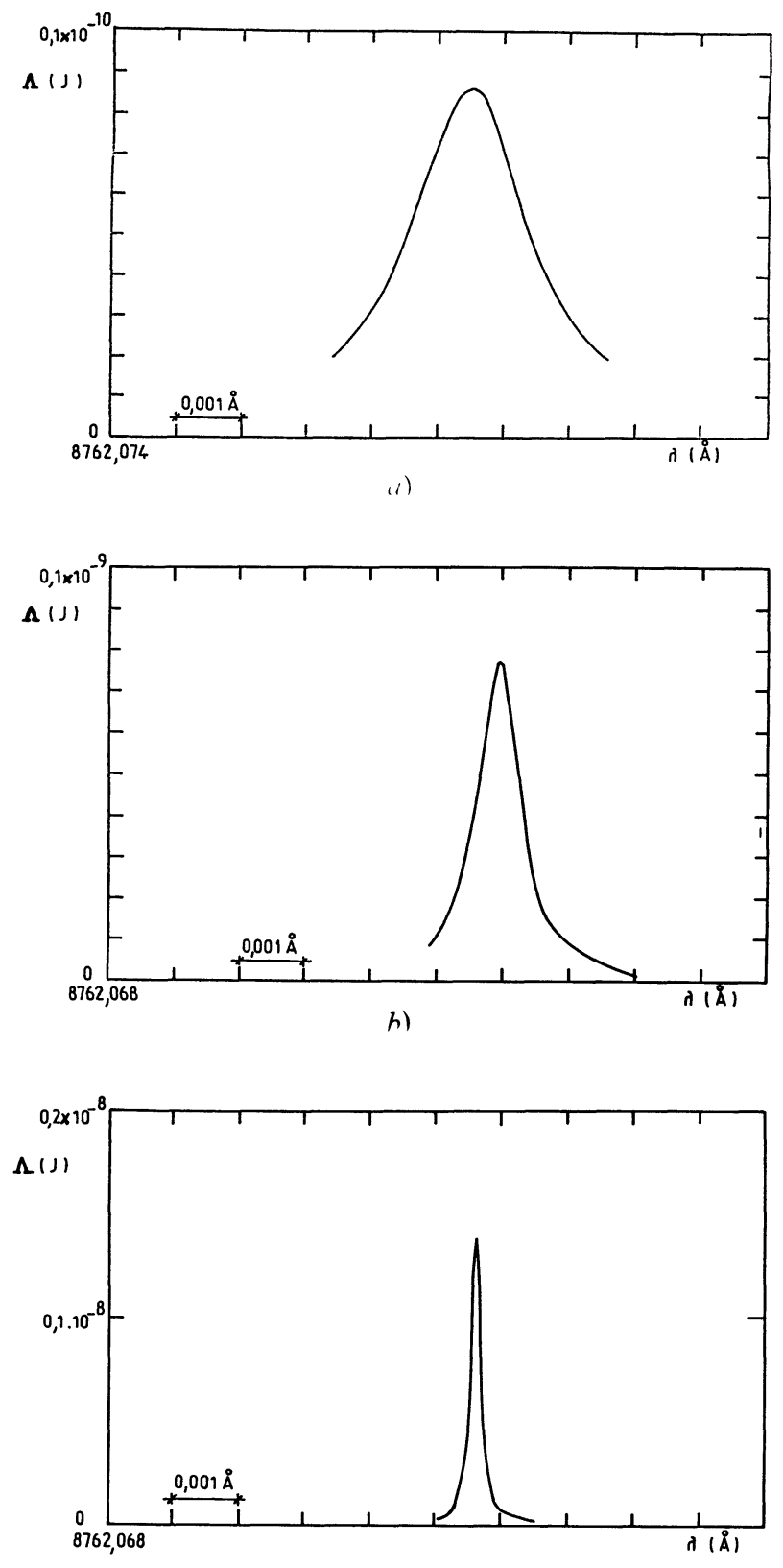

Fig. 14. - Spectres d'intensité du pic laser pour la structure long-court :

\begin{tabular}{lllll} 
& $\Delta E_{\mathrm{F} 2}(\mathrm{eV})$ & $I_{1}(\mathrm{~mA})$ & $I_{2}(\mathrm{~mA})$ & $P_{1}(\mathrm{~mW})$ \\
\cline { 2 - 5 } a & 1,448 & 14 & 13,6 & 0,43 \\
$\mathrm{~b}$ & 1,448 & 13,8 & 16,1 & 1,4 \\
$\mathrm{c}$ & 1,448 & 12,7 & 27,6 & 5,89
\end{tabular}

[Intensity spectra of the emitted peak for the short-long structure.]
$-\Delta f_{1}=87,9 \mathrm{MHz}$ correspondant à :

$$
\Delta \lambda=2,25 \times 10^{-3} \AA \quad P_{1}=0,43 \mathrm{~mW}
$$

$-\Delta f_{2}=29,3 \mathrm{MHz}$ correspondant à :

$$
\Delta \lambda=7,5 \times 10^{-4} \AA \quad P_{1}=1,4 \mathrm{~mW}
$$

$-\Delta f_{3}=6,642 \mathrm{MHz}$ correspondant à :

$$
\Delta \lambda=1,7 \times 10^{-4} \AA \quad P_{1}=5,89 \mathrm{~mW} .
$$

Ces résultats conduisent à une loi de variation en $\Delta \lambda=\alpha / P$ avec $\alpha=$ Cte. Dans le cas d'un laser conventionnel, Flemming et Moradien [20] ont trouvé expérimentalement une largeur de raie de $114 \mathrm{MHz}$ à $1 \mathrm{~mW}$ valeur qui a été expliquée théoriquement par C. Henry [21].

Notre modèle montre dans le cas de la structure laser à cavité couplée de type $\mathrm{C}^{3}$ présentée ici une largeur de raie théorique 3 à 4 fois plus faible que celle mesurée pour la même puissance sur un laser classique. Cependant, ce résultat est à confirmer avec un modèle plus rigoureux, prenant en compte la non-homogénéité des lignes de courant, les effets thermiques et l'influence de l'émission spontanée. Dans ce contexte, divers auteurs ont montré l'importance de la formulation du coefficient de répartition de l'émission spontanée sur les modes lasers, sur la largeur de raie $[24,25]$.

\section{Conclusion.}

Ce modèle constitue une étude théorique complète des structures lasers à cavités couplées relative à leur réponse en courant et en tension continus. Il prend en compte l'influence de l'injection des porteurs sur l'indice du milieu et traite de la structure réelle du guide de lumière à travers le calcul de l'indice effectif et du facteur de confinement.

Il permet d'expliquer le comportement général de ces structures. Basé sur un modèle d'amplificateur résonant prenant en compte la répartition de l'émission spontanée sur les modes lasers, il permet d'exprimer les intensités et les puissances lumineuses sur chaque miroir, ainsi que les spectres en puissance et en intensité.

L'exploitation faite du modèle dans la présente publication est relative au cas d'un laser à zone active GaAs. Sa formulation permet néanmoins de simuler différents cas, fonction de la température et du taux d'aluminium. 


\section{Bibliographie}

[1] Kuindersma, P. I., Binsma, J. J. M., Dijksterhuis, W., FREDRIKSZ, C. W., HOFSTAD, G. L. A. V. D., VAN Dongen, T., Ambrosius, H. P. M. M., 12th European Conference on Optical Communication. Technical Digest Vol. III, Barcelona (Sept. 1986), p. 53-56.

[2] Tsang, W. T., Olsson, N. A. and Logan, R. A., IEEE J. Quantum Electron QE-19 (1983) 162125.

[3] Temkin, H., VAn der Ziel, J. P., Linke, R. A. and Logan, R. A., Appl. Phys. Lett. 43 (1983) 723 25.

[4] Coldren, L. A., Miller, B. I., IgA, K. and Rentschler, J. A., Appl. Phys. Lett. 38 (1981) 315-17.

[5] Fleck, J. A. Jr., J. Appl. Phys. 34 (1963) 2997-3003.

[6] CHOI, H. K., Chen, K. L. and SHYH WANG, IEEE J. Quantum Electron QE-20 (1984) 385-93.

[7] ShyH WANG, Choi Hong, K. and FatTah Ismail, H. A., IEEE J. Quantum Electron QE-18 (1982) 610-17.

[8] Lozes, F., Thèse de Doctorat d'Etat, Université Paul Sabatier, Toulouse (1983) $\mathrm{n}^{\circ}$ d'ordre 1105.

[9] Kressel, H., Butler, J. K., Semiconductor Lasers and Heterojunction Leds (Academic Press, New York) 1977, p. 413.

[10] Manning, J., Olshansky, R. and Chin Bingsu, IEEE J. Quantum Electron QE-19 (1983) 152530.

[11] Casey, H. C. Jr., Panish, M. B., Heterostructure Lasers Part A (Academic Press, New York) 1978 , p. 45.
[12] Marcatilli, E. A. J., Bell Syst. Tech. J. 48 (1969) 2071.

[13] Kressel, H., Butler, J. K., Semiconductor Lasers and Heterojunction L.E.D.S. (Academic press, New York) 1977, p. 88.

[14] Henry, C. H., Logan, R. A., Bertness, K. A., J. Appl. Phys. 52 (1981) 4457.

[15] Peterman, K., IEEE J. Quantum Electron QE-15 (1979) 556-70.

[16] Tsang, W. T., Olson, N. A. and Logan, R. A., Appl. Phys. Lett. 42 (1983) 651-52.

[17] Streiffer, W., Yewick, D., PaOli, T. L., Burnam, R., IEEE J. Quantum Electron QE-20 (1984).

[18] MARCuSE and LeE, T. P., J. Quantum Electron QE20 (1984).

[19] Lee Tien-Pei, Burrus, C.H.A. Jr., Pao-Lo-Liu, SESSA, W. B. and LOGAN, R. A., IEEE J. of Quantum Electron QE-20 (1984).

[20] Fleming, M. W. and Mooradian, A., Appl. Phys. Lett. 38 (1981) 511.

[21] HENRY, C. H., IEEE J. of Quantum Electron. QE-18 (1982).

[22] Chоі, H. K. and Wang, S., Appl. Phys. Lett. 40 (1982) 571-73.

[23] ChOI, H. K. and WANG, S., Electron. Lett. 19 (1983) 302-303.

[24] Voumard, C., Salathe, R. and Weber, H., Appl. Phys. 12 (1977) 369-387.

[25] ARnaud, J., Electron. Lett. 21 (1985) 538-539. 